\title{
Mass Spectrum of $1{ }^{1} P_{1}$ Meson State and Mixing Angle of Strange Axial-Vector Mesons
}

\author{
Xue-Chao Feng, ${ }^{1}$ Ke-Wei Wei, ${ }^{2}$ Jie Wu, ${ }^{1}$ Yun-Qiang Zhang, \\ Ming-Yang $\mathrm{Wu}^{1}{ }^{1}$ and Feng-Chun Jiang ${ }^{1}$ \\ ${ }^{1}$ Department of Technology and Physics, Zhengzhou University of Light Industry, Zhengzhou 450002, China \\ ${ }^{2}$ College of Physics and Electrical Engineering, Anyang Normal University, Anyang 455002, China
}

Correspondence should be addressed to Xue-Chao Feng; fxchao@zzuli.edu.cn

Received 29 December 2012; Revised 6 March 2013; Accepted 26 March 2013

Academic Editor: Alexey Petrov

Copyright (C) 2013 Xue-Chao Feng et al. This is an open access article distributed under the Creative Commons Attribution License, which permits unrestricted use, distribution, and reproduction in any medium, provided the original work is properly cited.

In the presence of the $b_{1}(1235), h_{1}(1170), D_{1}(2420)$, and $D_{s 1}(2536)^{ \pm}$being the members of the $1{ }^{1} P_{1}$ meson multiplet, we investigate the mass spectrum of the $1^{1} P_{1}$ meson state and the mixing angle of strange axial-vector mesons in the framework of meson-meson mass mixing and Regge phenomenology. Also, in the glueball dominance picture, the kaon is phenomenologically determined to have a mass of about $1356 \mathrm{MeV}$. The results are compared with the values from different phenomenological models and may be useful for the assignment of the $1{ }^{1} P_{1}$ meson multiplet in the future.

\section{Introduction}

Quantum Chromodynamics (QCD) is widely accepted as a successful theory of the strong interactions in particle physics. However, the understanding of the strong interactions is far from complete, and it is difficult to interpret the particle properties for the experimental data from the first principles. To be able to determine the nature of a new experimental resonance, it is necessary to create a template to compare the observed states with theoretical predictions. Therefore, different phenomenological models, such as quenched lattice gauge theory [1], the Dyson-Schwinger formalism [2], and constituent quark model [3], are built to describe the properties of experimental results [4].

In the recent issue of Review of Particle Physics (PDG), the $b_{1}(1235), h_{1}(1170), D_{1}(2420)$, and $D_{s 1}(2536)^{ \pm}$have been observed in the experiment and assigned as the members of the $1^{1} P_{1}$ meson multiplet [5]. However, the assignment for the strange member $s \bar{s}$ state is problematic. On the one hand, based on the decay results in the ${ }^{3} P_{0}$ model, Barnes et al. suggest that the $h_{1}(1380)$ should be a candidate of the $1^{1} P_{1}$ meson [6]. On the other hand, the mass of $h_{1}(1380)$ is inconsistent with the prediction in the potential model, which is about $100 \mathrm{MeV}$ higher than the measured masses of the $h_{1}(1380)$ [7]. Accordingly, the mass and decays of the $h_{1}(1380)$ need to be further examined experimentally.

Apart from the $s \bar{s}$ member, the mass of the $K_{1 B}$ also remains interesting. Due to the states $K_{1}\left({ }^{1} P_{1}\right)$ and $K_{1}\left({ }^{3} P_{1}\right)$ having no definite C-parity and the $\mathrm{SU}(3)$ broken, the $K_{1}\left({ }^{1} P_{1}\right)$ and $K_{1}\left({ }^{3} P_{1}\right)$ can mix via the spin-orbit interaction to form the physical states $K_{1}(1270)$ and $K_{1}(1400)$. In order to compare the theoretical prediction with experimental data, the mixing angle of $K_{1 A}$ and $K_{1 B}$ has been discussed with different theoretical approaches [8-12]. In addition to the light meson states, the charmonium is one of the most exciting areas of high energy physics. In the $1^{1} P_{1}$ meson state, the $h_{c}(1 P)$ has been observed in the decay $\psi(2 s) \rightarrow \pi^{0} h_{c}$ with $h_{c} \rightarrow \gamma \eta_{c}$ by CLEO [13] and in the reaction $p \bar{p} \rightarrow h_{c} \rightarrow \gamma \eta_{c} \rightarrow \gamma \gamma \gamma$ [14]. The experimental signals about $h_{c}(1 P)$ are still very weak so far.

In allusion to the problems mentioned earlier, the purpose of this work is to investigate the mass spectrum of $1^{1} P_{1}$ meson state and mixing angle of strange axial-vector mesons. The results should be useful for exploring the nature and the assignment of $1^{1} P_{1}$ meson state. The organization of this paper is as follows. In Section 2, the brief review of the mass mixing matrix of isoscalar states is given (for 
the detailed review, see, e.g., [15-17]). In Section 3, the mass spectrum of $1^{1} P_{1}$ meson state is presented in the framework of Regge phenomenology and glueball-meson mass relation. The mixing angle of $K_{1 A}$ and $K_{1 B}$ is predicted in Section 4, and a summary and conclusion are given in Section 5.

\section{The Review of Mass Mixing Matrix of Isoscalar States}

In the quark model, the two isoscalar states with the same $J^{P C}$ quantum number will mix to form the physical states. One can obtain the mass-squared matrix in the $S=s \bar{s}$ and $N=$ $(u \bar{u}+d \bar{d}) / \sqrt{2}$ basis [18] as follows:

$$
M^{2}=\left(\begin{array}{cc}
M_{N}^{2}+2 A_{n n} & \sqrt{2} A_{n s} \\
\sqrt{2} A_{n s} & M_{s \bar{s}}^{2}+A_{s s}
\end{array}\right),
$$

where $M_{N}$ and $M_{s \bar{s}}$ are the masses of bare states $N$ and $S$, respectively; $A_{n n}, A_{n s}$, and $A_{s s}$ are the mixing parameters which describe the $q \bar{q} \leftrightarrow q^{\prime} \bar{q}^{\prime}$ transition amplitudes. In order to reduce the number of parameters, the following expressions are widely used in $[7,19]$ :

$$
A_{n s}=A_{n n} X, \quad A_{s s}=A_{n n} X^{2},
$$

where $X$ is a phenomenological parameter describing the $\mathrm{SU}(3)$ broken ratio of the nonstrange and strange quark propagators via the constituent quark mass ratio. In the present work, we take $X=0.63$, which is determined within the nonrelativistic constituent quark model $[17,20]$.

In the meson nonet, the physical isoscalar states $\varphi$ and $\varphi^{\prime}$ are the eigenstates of mass-squared matrix $M^{2}$, and the masses square $M_{\varphi}^{2}$ and $M_{\varphi^{\prime}}^{2}$ are the eigenvalues, respectively. The physical states $\varphi$ and $\varphi^{\prime}$ can be related to the $S=s \bar{s}$ and $N=(u \bar{u}+d \bar{d}) / \sqrt{2}$ by

$$
\left(\begin{array}{c}
|\varphi\rangle \\
\left|\varphi^{\prime}\right\rangle
\end{array}\right)=U\left(\begin{array}{c}
|N\rangle \\
|S\rangle
\end{array}\right),
$$

and the unitary matrix $U$ can be described as

$$
U M^{2} U^{\dagger}=\left(\begin{array}{cc}
M_{\varphi}^{2} & 0 \\
0 & M_{\varphi^{\prime}}^{2}
\end{array}\right) .
$$

The mix of the physical isoscalar states can also be described as

$$
\left(\begin{array}{l}
|\varphi\rangle \\
\left|\varphi^{\prime}\right\rangle
\end{array}\right)=\left(\begin{array}{cc}
\cos \theta & -\sin \theta \\
\sin \theta & \cos \theta
\end{array}\right)\left(\begin{array}{l}
\varphi_{8} \\
\varphi_{1}
\end{array}\right)
$$

with

$$
\begin{gathered}
\varphi_{1}=\frac{u \bar{u}+d \bar{d}+s \bar{s}}{\sqrt{3}}, \\
\varphi_{8}=\frac{u \bar{u}+d \bar{d}-2 s \bar{s}}{\sqrt{6}},
\end{gathered}
$$

where $\theta$ is the nonet mixing angle.
From the relations (3), (4), and (5), one will obtain

$$
U=\left(\begin{array}{cc}
\cos \theta & -\sin \theta \\
\sin \theta & \cos \theta
\end{array}\right)\left(\begin{array}{cc}
\sqrt{\frac{1}{3}} & -\sqrt{\frac{2}{3}} \\
\sqrt{\frac{2}{3}} & \sqrt{\frac{1}{3}}
\end{array}\right) .
$$

Based on the relations (4) and (7), the following relations are obtained:

$$
\begin{aligned}
M_{N}^{2}+2 A= & \left(\sqrt{\frac{1}{3}} \cos \theta-\sqrt{\frac{2}{3}} \sin \theta\right)^{2} M_{\varphi}^{2} \\
& +\left(\sqrt{\frac{2}{3}} \cos \theta+\sqrt{\frac{1}{3}} \sin \theta\right)^{2} M_{\varphi^{\prime}}^{2} \\
M_{s \bar{s}}^{2}+A X^{2}= & \left(\sqrt{\frac{1}{3}} \cos \theta-\sqrt{\frac{2}{3}} \sin \theta\right)^{2} M_{\varphi^{\prime}}^{2} \\
& +\left(\sqrt{\frac{2}{3}} \cos \theta+\sqrt{\frac{1}{3}} \sin \theta\right)^{2} M_{\varphi^{2}}^{2} .
\end{aligned}
$$

Considering the fact that $N$ is the orthogonal partner of the isovector state of a meson nonet, one can expect that $N$ degenerates with isovector state $M_{N}=M_{I=1}$. Applying the relations (8) for the ground pseudoscalar meson, we obtain the pure $s \bar{s}$ member mass of the ground pseudoscalar meson nonet $M_{s \bar{s}}\left(1^{1} S_{0}\right)=685 \mathrm{MeV}$ [17]. Here and in the following, all the masses used as input for our calculation are taken from the PDG [5].

\section{Mass Spectrum of $1^{1} P_{1}$ Meson State in Regge Phenomenology and Glueball Dominance Picture}

Regge theory is cornered with the particle spectrum, the forces between particles, and the high energy behavior of scattering amplitudes. In [21], Li et al. investigate the masses of different meson multiplets in the framework of Regge phenomenology and suggest that the quasilinear Regge trajectories could provide a reasonable description for the meson mass spectrum. Khruschov predicts the masses spectrum of excited meson states using the phenomenology formulas derived from the Regge trajectories [22]. Anisovich et al. also suggest that the mass of meson states can fit to the quasilinear Regge trajectories with good accuracy [23]. In the last decade, Regge trajectories are reanalysed to make predictions for the masses of states not yet to be discovered in experiment and to determine the quantum numbers of the newly discovered states [21-28].

Based on the assumption that the hadrons with identical $J^{P C}$ quantum numbers obey quasilinear form of Regge trajectories, one has the following relations [21]:

$$
\begin{aligned}
& l=\alpha_{\overline{i i^{\prime}}}(0)+\alpha_{\overline{i^{\prime}}}^{\prime} M_{\overline{i i^{\prime}}}^{2}, \\
& l=\alpha_{i \overline{j^{\prime}}}(0)+\alpha_{i \bar{j}^{\prime}}^{\prime} M_{i \overline{j^{\prime}}}^{2}, \\
& l=\alpha_{j \overline{j j^{\prime}}}(0)+\alpha_{j \overline{j^{\prime}}}^{\prime} M_{j j^{\prime}}^{2},
\end{aligned}
$$


TABLE 1: Comparison of predictions for the masses of the $1^{1} P_{1}$ meson states with other references. (All in MeV.)

\begin{tabular}{lccccccc}
\hline & Present work & {$[5]$} & {$[15]$} & {$[7]$} & {$[31]$} & {$[32]$} & {$[33]$} \\
\hline$M_{s \bar{s}}$ & 1499 & 1386 & 1495.18 & 1470 & & & \\
$M_{n \bar{s}}$ & 1375 & & 1370.03 & 1340 & 1368 & 1356 & \\
$M_{c \bar{c}}$ & 3509 & 3525.93 & & 3520 & & & 3526 \\
\hline
\end{tabular}

where $i \bar{i}$ denotes the quark and antiquark flavors, and $l$ and $M_{i i^{\prime}}$ are the orbital momentum and mass of the $\overline{i i^{\prime}}$ meson. The parameters $\alpha_{i i^{\prime}}^{\prime}$ and $\alpha_{i i^{\prime}}(0)$ are, respectively, the slope and intercept of the Regge trajectory. The intercepts can be described as the following relation by $[21,24]$ :

$$
\alpha_{i \bar{i}}(0)+\alpha_{j \bar{j}}(0)=2 \alpha_{i \bar{j}}(0) .
$$

The relation (10) is satisfied in two-dimensional QCD [29] and the quark bremsstrahlung model [30].

With the aid of the parity partners trajectories having the same slopes, we can apply relations (9)-(10) to the $1^{1} S_{0}$ and ${ }_{1}^{1} P_{1}$ meson multiplets to predict the following meson masses:

$$
\begin{aligned}
& \alpha_{n \bar{n}}^{\prime} M^{2}\left(1^{1} S_{0}\right)_{n \bar{n}}+\alpha_{s \bar{s}}^{\prime} M^{2}\left(1^{1} S_{0}\right)_{s \bar{s}}=2 \alpha_{n \bar{s}}^{\prime} M^{2}\left(1^{1} S_{0}\right)_{n \bar{s}} \\
& \alpha_{n \bar{n}}^{\prime} M^{2}\left(1^{1} S_{0}\right)_{n \bar{n}}+\alpha_{c \bar{c}}^{\prime} M^{2}\left(1^{1} S_{0}\right)_{\bar{c}}=2 \alpha_{c \bar{n}}^{\prime} M^{2}\left(1^{1} S_{0}\right)_{\bar{n} \bar{n}} \\
& \alpha_{s \bar{s}}^{\prime} M^{2}\left(1^{1} S_{0}\right)_{s \bar{s}}+\alpha_{c \bar{c}}^{\prime} M^{2}\left(1^{1} S_{0}\right)_{c \bar{c}}=2 \alpha_{c \bar{s}}^{\prime} M^{2}\left(1^{1} S_{0}\right)_{c \bar{s}}, \\
& \alpha_{n \bar{n}}^{\prime} M^{2}\left(1^{1} P_{1}\right)_{n \bar{n}}+\alpha_{\bar{s} \bar{s}}^{\prime} M^{2}\left(1^{1} P_{1}\right)_{s \bar{s}}=2 \alpha_{n \bar{s}}^{\prime} M^{2}\left(1^{1} P_{1}\right)_{n \bar{s}}, \\
& \alpha_{n \bar{n}}^{\prime} M^{2}\left(1^{1} P_{1}\right)_{n \bar{n}}+\alpha_{c \bar{c}}^{\prime} M^{2}\left(1^{1} P_{1}\right)_{c \bar{c}}=2 \alpha_{\bar{c} \bar{n}}^{\prime} M^{2}\left(1^{1} P_{1}\right)_{c \bar{n}} \\
& \alpha_{\bar{s} \bar{s}}^{\prime} M^{2}\left(1^{1} P_{1}\right)_{s \bar{s}}+\alpha_{c \bar{c}}^{\prime} M^{2}\left(1^{1} P_{1}\right)_{c \bar{c}}=2 \alpha_{c \bar{s}}^{\prime} M^{2}\left(1^{1} P_{1}\right)_{c \bar{s}} \cdot
\end{aligned}
$$

In order to eliminate the Regge slopes from the previous formulas, we adopt the following relation [24]:

$$
\frac{1}{\alpha_{\overline{i i^{\prime}}}^{\prime}}+\frac{1}{\alpha_{j j^{\prime}}^{\prime}}=\frac{2}{\alpha_{i j^{\prime}}^{\prime}} \text {. }
$$

For the $1^{1} S_{0}$ meson multiplet, all members are established well. For the $1^{1} P_{1}$ meson multiplet, only the $b_{1}(1235)$ and $h_{1}(1170)$ are established well, and the strange meson $K_{1 B}$ and the charmed mesons $D_{1}(2420)$ and $D_{s 1}(2536)^{ \pm}$are the mixtures of spin-singlet $1^{1} P_{1}$ and spin-triplet $1^{3} P_{1}$ states. In the present work, based on the small mass splitting between charmed mesons, we conclude that the effect of mixing on the charmed meson masses will be small. Therefore, inserting the masses of the corresponding mesons states and $M\left(1^{1} P_{1}\right)_{\overline{c s}}=$ $M_{D_{s 1}(2536)}$ as well as $M\left(1^{1} P_{1}\right)_{\bar{c} \bar{n}}=M_{D_{1}(2420)}$ into relations (11)(12), the masses of the $s \bar{s}$ member, $K_{1 B}$, and $h_{c}(1 P)$ for the $1^{1} P_{1}$ meson multiplet are obtained, and the results are shown in Table 1.

The mass of the $K_{1 B}$ is important for the calculation of mixing angle of the $K_{1 A}$ and $K_{1 B}$ states. In order to check consistence of the results in Table 1, we investigate the mass of kaon in the glueball dominance picture in the next section [18]. And the result is in good agreement with the prediction in the Regge phenomenology.
TABLE 2: Masses (in GeV) of the ground tensor and pseudovector glueball from different theoretical models.

\begin{tabular}{lcccccccc}
\hline Mass & {$[34]$} & {$[35]$} & {$[36]$} & {$[37]$} & {$[38]$} & {$[39]$} & {$[40]$} & Average \\
\hline$M_{\text {ten }}$ & 2.59 & 2.40 & 2.39 & 2.15 & 2.26 & 2.4 & 2.417 & 2.37 \\
$M_{\text {pse }}$ & & 2.94 & 2.98 & 2.67 & 2.71 & & & 2.83 \\
\hline
\end{tabular}

In the mass matrix, the quark mixing amplitudes in (1) can be expressed as

$$
A_{q q^{\prime}}=\sum_{k} \frac{\left\langle q \bar{q}\left|H_{p . c .}^{q^{\prime} \bar{q}^{\prime}}\right| k\right\rangle\left\langle k\left|H_{p . c .}^{q \bar{q}}\right| q^{\prime} \overline{q^{\prime}}\right\rangle}{M_{q^{\prime} \bar{q}^{\prime}}^{2}-M_{k}^{2}}
$$

where $H_{p . c .}^{q \bar{q}}$ is the quark pair creation operator for the flavor $q$, and $|k\rangle$ is a complete set of the intermediate states. Based on the assumption that there is no direct quarkoniumquarkonium mixing and the $q \bar{q} \leftrightarrow q^{\prime} \overline{q^{\prime}}$ transitions are dominated by the ground glueball with the corresponding quantum numbers, we obtain the following relations:

$$
\begin{aligned}
A_{n n} & =\frac{f_{n \bar{n} G}^{2}}{M_{n n}^{2}-M_{G}^{2}}, \\
A_{s s} & =\frac{f_{s \bar{s} G}^{2}}{M_{s s}^{2}-M_{G}^{2}},
\end{aligned}
$$

where $\left.f_{q \bar{q} G} \equiv\left\langle q \bar{q}\left|H^{q \bar{q}}\right| G\right\rangle\right|_{p^{\mu} P_{\mu}=M_{q \bar{q}}^{2}}$ and $q$ refers to the $n$ and $s$ quarks. The parameters $M_{n n}^{2}=M_{I=1}^{2}$ and $M_{s s}^{2}=2 M_{I=1 / 2}^{2}-$ $M_{I=1}^{2} ; M_{I=1}$ and $M_{I=1 / 2}$ are the masses of the isovector and isodoublet states of a meson nonet. Considering the functions $f_{q \bar{q} G}$, Brisudova et al. indicate the product of $f_{\bar{n} \bar{n} G}$ and $f_{s \bar{s} G}$ in QCD to be a constant approximately independent of the quantum numbers of a meson nonet; namely [18],

$$
f_{n \bar{n} G} f_{s \bar{s} G} \approx \text { Const. }
$$

Following the relations (1), (4), and (14)-(15), we have

$$
\frac{\left(M_{b_{1}(1235)}^{2}-M_{\mathrm{pse}}^{2}\right)\left(2 M_{K_{1 B}}^{2}-M_{b_{1}(1235)}^{2}-M_{\mathrm{pse}}^{2}\right)}{\left(M_{a_{2}(1320)}^{2}-M_{\mathrm{ten}}^{2}\right)\left(2 M_{K_{2}^{*}}^{2}-M_{a_{2}(1320)}^{2}-M_{\mathrm{ten}}^{2}\right)}=\frac{A_{1^{3} P_{2}}}{A_{1^{1} P_{1}}}
$$

with

$$
\begin{aligned}
& A_{1^{1} P_{1}}=\frac{M_{h_{1}(1170)}^{2}+M_{h_{1}(1380)}^{2}-2 M_{K_{1 B}^{2}}^{2},}{2+X^{2}}, \\
& A_{1^{3} P_{2}}=\frac{M_{f_{2}(1270)}^{2}+M_{f_{2}^{\prime}(1525)}^{2}-2 M_{K_{2}^{*}}^{2}}{2+X^{2}},
\end{aligned}
$$

where $M_{\text {ten }}$ and $M_{\text {pse }}$ are the masses of ground tensor and pseudovector glueball, respectively. In order to extract the mass of $K_{1 B}$ by using relation (16), we should determine the approximate masses of ground tensor and pseudovector glueballs. Some predictions on the masses of the glueball given by different theoretical models are shown in Table 2. In the present work, taking the masses of corresponding mesons in the relation (16) and the average values of glueball masses in Table 2 as input, we obtain the mass of the $K_{1 B}$. Our predicted result $1356 \mathrm{MeV}$ is in agreement with our previous work. 
TABLE 3: Predicted mixing angle $\phi$ of the $K_{1}(1270)$ and $K_{1}(1400)$.

\begin{tabular}{|c|c|c|c|c|c|c|c|}
\hline Refs & Present work & [15] & [12] & [41] & {$[42]$} & {$[43]$} & {$[44]$} \\
\hline$\overline{|\phi|}$ & $61.6^{\circ}$ & $(59.55 \pm 2.81)^{\circ}$ & $59.29^{\circ}$ & $54.7^{\circ}$ or $35.3^{\circ}$ & $62^{\circ}$ & $55.7^{\circ}$ & $60^{\circ}$ \\
\hline
\end{tabular}

\section{The Mixing Angle of $K_{1 A}$ and $K_{1 B}$}

According to the introduction in Section 1, the $K_{1 A}$ and $K_{1 B}$ in the $1^{3} P_{1}$ and $1^{1} P_{1}$ meson states can mix to produce the physical states $K_{1}(1270)$ and $K_{1}(1400)$. In the $\left|1^{1} P_{1}\right\rangle$ and $\left|1^{3} P_{1}\right\rangle$ basis, the physical states $K_{1}(1270)$ and $K_{1}(1400)$ can be parameterized as [11]

$$
\begin{aligned}
& K_{1}(1270)=\sin \phi\left|1^{3} P_{1}\right\rangle+\cos \phi\left|1^{1} P_{1}\right\rangle, \\
& K_{1}(1400)=\cos \phi\left|1^{3} P_{1}\right\rangle-\sin \phi\left|1^{1} P_{1}\right\rangle .
\end{aligned}
$$

In this work, $\phi$ is defined as the $K_{1 A}$ and $K_{1 B}$ mixing angle. To obtain the mixing angle $\phi$, we use the formula [31]

$$
\tan ^{2}(2 \phi)=\left(\frac{M_{K_{1 A}}^{2}-M_{K_{1 B}}^{2}}{M_{K_{1}(1400)}^{2}-M_{K_{1}(1270)}^{2}}\right)^{2}-1
$$

The relation (19) can also be rewritten as

$$
\cos (2 \phi)=\frac{M_{K_{1}(1400)}^{2}-M_{K_{1}(1270)}^{2}}{M_{K_{1 A}}^{2}-M_{K_{1 B}}^{2}}
$$

Moreover, using the following relation which is independent of the mixing angle, the mass of $K_{1 A}$ is determined to be $1303 \mathrm{MeV}$. Consider

$$
M_{K_{1 A}}^{2}+M_{K_{1 B}}^{2}=M_{K_{1}(1400)}^{2}+M_{K_{1}(1270)}^{2} .
$$

With the help of the masses of $K_{1 A}, K_{1 B}, K_{1}(1270)$, and $K_{1}(1400)$, the mixing angle is determined to be $\pm 61.6^{\circ}$.

\section{Results and Conclusions}

Based on the fact that the $b_{1}(1235), h_{1}(1170), D_{1}(2420)$, and $D_{s 1}(2536)^{ \pm}$are assigned as the $1^{1} P_{1}$ meson multiplet, we obtain the masses of the $K_{1 B}, h_{1}(1380)$, and $h_{c}(1 P)$ in the ${ }^{1} P_{1}$ meson state. The mass of the $h_{1}(1380)$ is determined to be $1492 \mathrm{MeV}$, which is consistent with the value $1470 \mathrm{MeV}$ predicted by Godfrey and Isgur in the potential model [7]. The value is about $100 \mathrm{MeV}$ higher than the measured mass of the $h_{1}(1380)$ in the experiment. Besides, experimental information on the $h_{1}(1380)$ is rather restricted, which has been reported only by two collaborations, LASS Collaboration [45] (with mass $1380 \pm 20 \mathrm{MeV}$ and width $80 \pm 30 \mathrm{MeV}$ ) and Crystal Barrel Collaboration [46] (with mass $1440 \pm 60 \mathrm{MeV}$ and width $170 \pm 80 \mathrm{MeV}$ ). Therefore, we suggest that the assignment for this state needs to be further tested in the new experiments in the future.

The mass of $K_{1 B}$ is useful for determining the mixing angle of the $K_{1}(1270)$ and $K_{1}(1400)$. In the present work, the mass for $K_{1 B}$ is determined to be $1375 \mathrm{MeV}$, which is in agreement with the value from the meson-glueball mass relation and other theoretical approaches. The mixing angle of $K_{1}(1270)$ and $K_{1}(1400)$ is compared with other different predictions in Table 3. Our predictions may open a window for the assignment of the $1^{1} P_{1}$ meson multiplet in the new experiment in the future.

\section{Acknowledgments}

This project is supported by the Zhengzhou University of Light Industry Foundation of China (Grant nos. 2009XJJ011 and 2012XJJ008), in part by the National Natural Science Foundation of China (Grant nos. 10975018 and 11147197), and the Key Project of Scientific and Technological Research of the Education Department of Henan Province (Grant nos. 12B140001 and 13B140332).

\section{References}

[1] A. M. Polyakov, "Interaction of goldstone particles in two dimensions. Applications to ferromagnets and massive YangMills fields," Physics Letters B, vol. 59, no. 1, pp. 79-81, 1975.

[2] P. Maris and C. D. Roberts, "Dyson-schwinger equations: a tool for hadron physics," International Journal of Modern Physics E, vol. 12, no. 3, article 297, 2003.

[3] M. Gell-Mann, "A schematic model of baryons and mesons," Physics Letters, vol. 8, no. 3, pp. 214-215, 1964.

[4] X. C. Feng, Y. Jia, T. Q. Chang, and J. Y. Li, "Radial excitation mass spectrum of tensor meson nonet," Acta Physica Polonica $B$, vol. 42, no. 1, pp. 25-32, 2011.

[5] J. Beringer, J. F. Arguin, R. M. Barnett et al., "Review of particle physics," Physical Review D, vol. 86, no. 1, Article ID 010001, 2012.

[6] T. Barnes, N. Black, and P. R. Page, "Strong decays of strange quarkonia," Physical Review D, vol. 68, no. 5, Article ID 054014, 43 pages, 2003.

[7] S. Godfrey and N. Isgur, "Mesons in a relativized quark model with chromodynamics," Physical Review D, vol. 32, no. 1, pp. 189-231, 1985.

[8] N. Isgur and M. B. Wise, "Weak decays of heavy mesons in the static quark approximation," Physics Letters B, vol. 232, no. 1, pp. 113-117, 1989.

[9] D. M. Asner, A. Eppich, J. Gronberg et al., "Resonance structure of $\tau^{-} \rightarrow K^{-} \pi^{+} \pi^{-} v_{\tau}$," Physical Review D, vol. 62, no. 7, Article ID 072006, 11 pages, 2000.

[10] H. Y. Cheng, "Hadronic charmed meson decays involving axial vector mesons," Physical Review D, vol. 67, no. 9, Article ID 094007, 10 pages, 2003.

[11] M. Suzuki, "Strange axial-vector mesons," Physical Review D, vol. 47, no. 3, pp. 1252-1255, 1993.

[12] D. M. Li and Z. Li, "Strange axial-vector mesons mixing angle," The European Physical Journal A, vol. 28, pp. 369-373, 2006.

[13] J. L. Rosner, N. E. Adam, J. P. Alexander et al., "Observation of the $h_{c}\left({ }^{1} P_{1}\right)$ state of charmonium," Physical Review Letters, vol. 95, no. 10, Article ID 102003, 10 pages, 2005. 
[14] M. Andreotti, S. Bagnasco, W. Baldini et al., "Results of a search for the $h_{c}\left({ }^{1} P_{1}\right)$ state of charmonium in the $\eta_{c} \gamma$ and $J / \psi \pi^{0}$ decay modes," Physical Review D, vol. 72, no. 3, Article ID 032001, 11 pages, 2005.

[15] D. M. Li, B. Ma, and Y. Hong, "Regarding the axial-vector mesons," The European Physical Journal A, vol. 26, pp. 141-145, 2005.

[16] X. C. Feng and J. L. Feng, "The mass of the isodoublet of the $2^{1}$ $S_{0}$ meson nonet revisited," Chinese Physics, vol. 16, no. 11, pp. 3297-3299, 2007.

[17] X. C. Feng, P. Chen, and J. M. Shang, "Towards the assignment for the ground scalar meson," Advances in High Energy Physics, vol. 2013, Article ID 219456, 7 pages, 2013.

[18] M. M. Brisudova, L. Burakovsky, and T. Goldman, "New glueball-meson mass relations," Physical Review D, vol. 58, no. 11, Article ID 114015, 7 pages, 1998.

[19] I. Cohen and H. J. Lipkin, "A phenomenological model for pseudoscalar meson mixing," Nuclear Physics B, vol. 151, pp. 1628, 1979.

[20] D. M. Li, K. W. Wei, and H. Yu, "A possible assignment for the ground scalar meson nonet," The European Physical Journal A, vol. 25, no. 2, pp. 263-266, 2005.

[21] D. M. Li, B. Ma, Y. X. Li, Q. K. Yao, and H. Yu, "Meson spectrum in Regge phenomenology," The European Physical Journal C, vol. 37, no. 3, pp. 323-333, 2004.

[22] V. V. Khruschov, "Mass spectra of excited meson states consisting of u-, d-quarks and antiquarks," http://arxiv.org/abs/hep$\mathrm{ph} / 0504077$.

[23] A. V. Anisovich, V. V. Anisovich, and A. V. Sarantsev, "Systematics of $q \bar{q}$ states in the $\left(n, M^{2}\right)$ and $\left(J, M^{2}\right)$ planes," Physical Review D, vol. 62, no. 5, Article ID 051502, 5 pages, 2000.

[24] L. Burakovsky and T. Goldman, "On the Regge slopes intramultiplet relation," Physics Letters B, vol. 434, no. 3-4, pp. 251-256, 1998.

[25] M. M. Brisudova, L. Burakovsky, and J. T. Goldman, "Effective functional form of Regge trajectories," Physical Review D, vol. 61, no. 5, Article ID 054013, 15 pages, 2000.

[26] Z. C. Ye, X. Wang, X. Liu, and Q. Zhao, "The mass spectrum and strong decays of isoscalar tensor mesons," Physical Review $D$, vol. 86, no. 5, Article ID 054025, 14 pages, 2012.

[27] P. Masjuan, E. R. Arriola, and W. Broniowski, "Systematics of radial and angular-momentum Regge trajectories of light nonstrange $q \bar{q}$-states," Physical Review D, vol. 85 , no. 9, Article ID 094006, 15 pages, 2012.

[28] E. R. Arriola and W. Broniowski, "Scalar-isoscalar states in the large- $N_{c}$ Regge approach,” Physical Review D, vol. 81, no. 5, Article ID 054009, 18 pages, 2010.

[29] R. C. Brower, J. Ellis, M. G. Schmidt, and J. H. Weis, "What is the relativistic generalization of a linearly rising potential?" Nuclear Physics B, vol. 128, no. 1, pp. 75-92, 1977.

[30] V. V. Dixit and L. A. P. Balázs, "Regge intercepts of multiquark systems in Feynman's bremsstrahlung analogy," Physical Review $D$, vol. 20, no. 3, pp. 816-819, 1979.

[31] P. V. Chliapnikov, " $S$ - and $P$-wave meson spectroscopy in the nonrelativistic quark model," Physics Letters B, vol. 496, no. 3-4, pp. 129-136, 2000.

[32] L. Burakovsky and T. Goldman, "Regarding the enigmas of $P$ wave meson spectroscopy," Physical Review D, vol. 57, no. 5, pp. 2879-2888, 1998.

[33] D. Ebert, R. N. Faustov, and V. O. Galkin, "Properties of heavy quarkonia and $B_{c}$ mesons in the relativistic quark model,"
Physical Review D, vol. 67, no. 1, Article ID 014027, 19 pages, 2003.

[34] A. B. Kaidolv and A. Yu. Simonov, "Glueball masses and Pomeron trajectory in nonperturbative QCD approach," Physics Letters B, vol. 477, no. 1-3, pp. 163-170, 2000.

[35] C. J. Morningstar and M. Peardon, "Glueball spectrum from an anisotropic lattice study," Physical Review D, vol. 60, no. 3, pp. 1-13, 1999.

[36] Y. Chen, A. Alexandru, and S. J. Dong, "Glueball spectrum and matrix elements on anisotropic lattices," Physical Review D, vol. 73, no. 1, Article ID 014516, 21 pages, 2006.

[37] H. B. Meyer, "Glueball regge trajectories," http://arxiv.org/ abs/hep-lat/0508002.

[38] M. J. Temper, "Physics from the lattice: glueballs in QCD; topology; SU(N) for all N," NATO Science B, vol. 368, pp. 4374, 2002.

[39] C. Liu, "A lattice study of the glueball spectrum," Chinese Physics Letters, vol. 18, no. 2, pp. 187-189, 2001.

[40] D. Q. Liu, J. M. Wu, and Y. Chen, "New approach to constructing the operator on the lattice for the calculation of the glueball masses," High Energy Physics and Nuclear Physics, vol. 26, no. 3, pp. 222-229, 2002.

[41] N. Isgur and M. B. Wise, "Weak decays of heavy mesons in the static quark approximation," Physics Letters B, vol. 232, no. 1, pp. 113-117, 1989.

[42] J. Rosner, " $P$ wave mesons with one heavy quark," Comments Nuclear and Particle Physics, vol. 16, article 109, 1986.

[43] J. Vijande, F. Fernández, and A. Valcarce, "Constituent quark model study of the meson spectra," Journal of Physics G, vol. 31, no. 5 , article 481, 2005.

[44] A. Tayduganov, E. Kou, and A. Le Yaouanc, "The strong decays of $K_{1}$ resonances," Physical Review D, vol. 85, no. 7, Article ID 074011, 28 pages, 2012.

[45] D. Aston, N. Awaji, T. Bienz et al., "Evidence for two strangeonium resonances with $J^{P C}=1^{++}$and $1^{+-}$in $K^{-} p$ interactions at $11 \mathrm{GeV} /$ c," Physics Letters B, vol. 201, no. 4, pp. 573-578, 1998.

[46] A. Abel, J. Adomeit, C. Amsler et al., "Antiproton-proton annihilation at rest into $K_{L} K_{S} \pi^{0} \pi^{0}$," Physics Letters B, vol. 415, no. 3, pp. 280-288, 1997. 

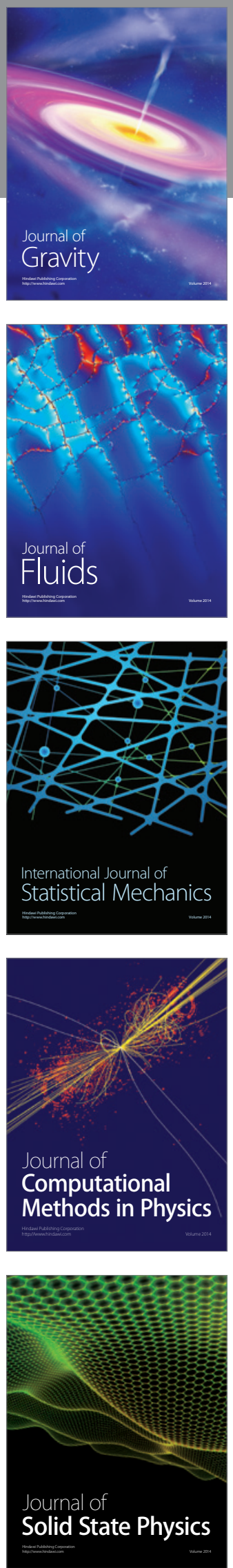

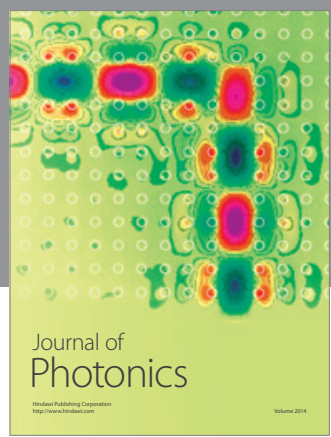

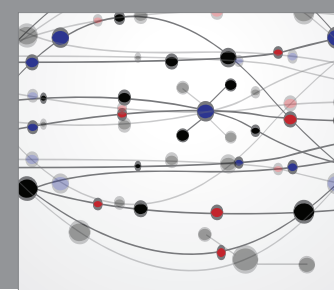

The Scientific World Journal

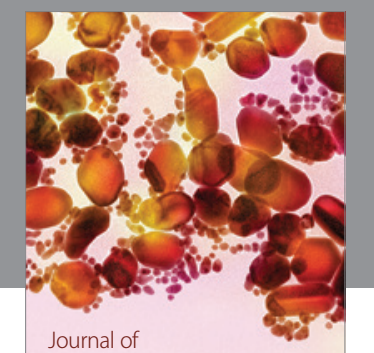

Soft Matter
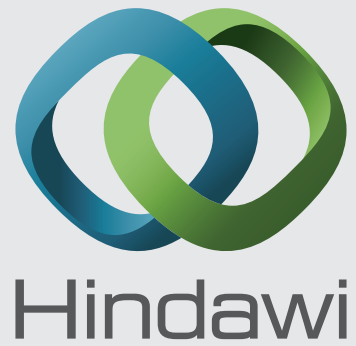

Submit your manuscripts at

http://www.hindawi.com
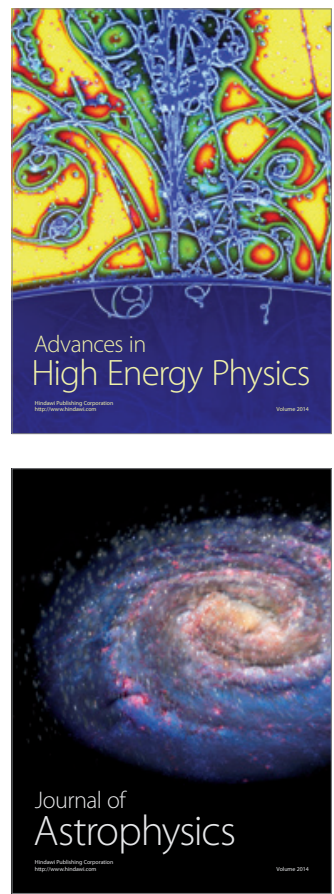
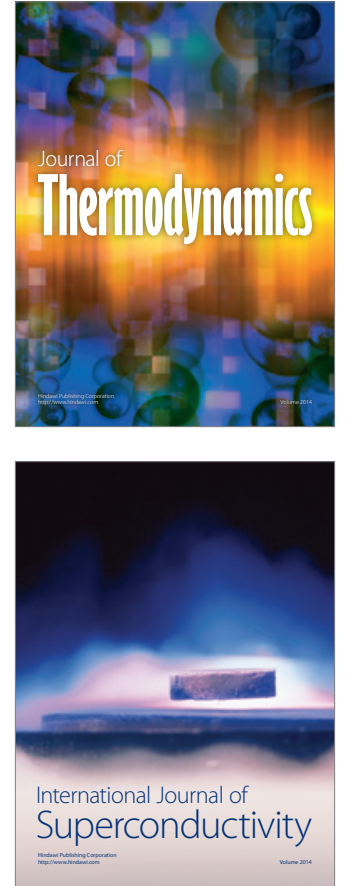
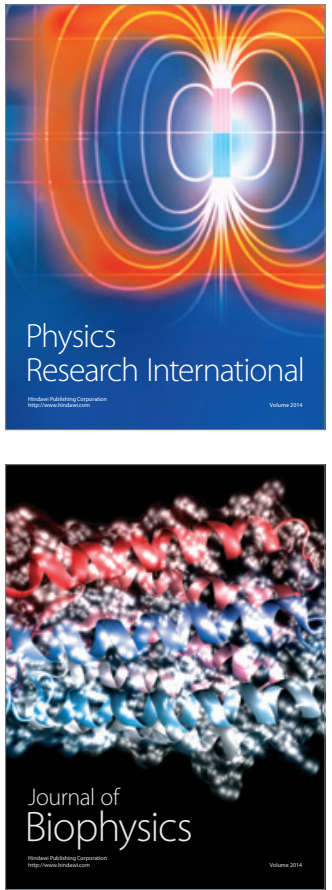
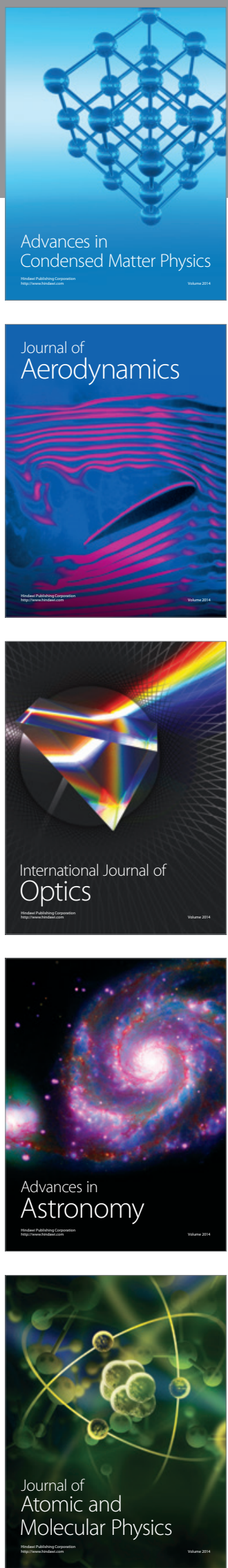